\title{
Effects of electroacupuncture at different acupoints on the histomorphology of neurogenic bladder and the expression of hyperpolarization-activated cyclic nucleotide-gated channels in interstitial cells of Cajal in a rat model of suprasacral spinal cord injury
}

\author{
Jun-Yan Lu, Xin-Wang Ying, Xiao-Long Chen, Wen-Zhan Tu, Si-Si Li, Song-He Jiang \\ Department of Physical Medicine \& Rehabilitation, The Second Affiliated Hospital of Wenzhou Medical University, Wenzhou, China \\ Contributions: (I) Conception and design: JY Lu; (II) Administrative support: XW Ying; (III) Provision of study materials or patients: XL Chen; (IV) \\ Collection and assembly of data: WZ Tu; (V) Data analysis and interpretation: SH Jiang; (VI) Manuscript writing: All authors; (VII) Final approval of \\ manuscript: All authors. \\ Correspondence to: Song-He Jiang. The Second Affiliated Hospital of Wenzhou Medical University, No. 109 West Academy Road, Lucheng District, \\ Wenzhou 325000, China. Email: jshwz@126.com.
}

\begin{abstract}
Background: To investigate the effects of electroacupuncture at different acupoints on the histomorphology of neurogenic bladder and the expression of hyperpolarization-activated cyclic nucleotidegated (HCN) channels in interstitial cells of Cajal (ICC) in a rat model of suprasacral spinal cord injury (SCI). Methods: A incomplete suprasacral SCI rat model was induced using a MASCIS impactor. Rats were randomly divided into a sham operation group, SCI model group, Ciliao treatment group or Guanyuan treatment group. The histomorphology of bladder cells was observed after hematoxylin and eosin (H\&E) staining of bladder tissue sections. The expression of HCN channel proteins in ICC cells was detected by western blot and immunofluorescence, and HCN channel mRNA expression was measured using real-time PCR.

Results: In terms of histomorphology, the level of bladder cells after SCI increased significantly, and marked inflammation and edema were observed. Electroacupuncture treatment at the Ciliao acupoint significantly reduced inflammation and edema, whilst electroacupuncture treatment at the Guanyuan point partially reduced inflammation and edema. In terms of HCN channel protein and mRNA expression, western blotting, immunofluorescence and real-time PCR all confirmed that HCN channel expression after SCI was significantly upregulated, while electroacupuncture treatment at the Ciliao and Guanyuan acupoints inhibited HCN channel expression.
\end{abstract}

Conclusions: Electroacupuncture treatment at the Ciliao acupoint significantly reduced histomorphological abnormalities in ICCs, and inhibited the expression of HCN channel proteins after SCI.

Keywords: Spinal cord injury; electroacupuncture; acupoints; neurogenic bladder; hyperpolarization-activated cyclic nucleotide-gated

Submitted Aug 10, 2020. Accepted for publication Nov 13, 2020.

doi: 10.21037/apm-20-1827

View this article at: http://dx.doi.org/10.21037/apm-20-1827 


\section{Introduction}

In recent years, the number of patients with spinal cord injury (SCI) has gradually increased. Most acute SCI is caused by traumatic vertebral compression or fracture dislocation. The acute stage of SCI leads to an interruption of the coordination function of the brain's micturition center, causing detrusor sphincter coordination disorder to occur during micturition $(1,2)$. When the detrusor contracts, the urethral sphincter continues to contract instead of relaxing, resulting in a large amount of residual urine. The urethral sphincter acts as a medium for bacterial growth, causing urinary tract infections, which in turn cause pyelonephritis and even kidney failure and death $(3,4)$. Therefore, recovery of bladder function is a major concern for all patients with SCI and their doctors. However, for various reasons, research on the treatment of this disease is lacking. There are currently no reliable drugs or treatment strategies. Intermittent catheterization is prone to urinary infections. Surgery, such as extracorporeal sphincter resection, pudendal nerve resection, spinal nerve root resection and small intestine cystoplasty, can have complex risks which patients find difficult to accept. In contrast, traditional acupuncture treatment is safe, convenient and does not have side effects, making it suitable for promoted use (5). Relevant research shows that acupuncture and moxibustion therapy has good curative effect on a series of complications and sequelae caused by spinal cord injury (6-9).

Interstitial cells of Cajal (ICCs) were first discovered in 1893 and have been shown to act as pacemakers, involved in generating slow wave activity and driving gastrointestinal peristalsis (10). HCN channels include 4 subtypes (HCN1-4) in mammals, and produce hyperpolarization-activated currents (Ih). HCN channels are involved in a variety of systemic functions, such as cardiac contractility, hormonal regulation, central pattern generation, sensory perception, and learning and memory (11-13). In previous studies, we detected that all $4 \mathrm{HCN}$ subtypes were expressed only in bladder ICCs, with the HCN1 channel being the most important one. HCN channels in ICCs are thought to be involved in the control of bladder pacemaker activity (14). A large body of evidence suggests that altered HCN pathways are associated with multiple excitatory diseases, such as various types of epilepsy, sinus bradycardia, atrial fibrillation, and Hirschsprung's disease (15-18). However, the role of HCN channels in bladder ICCs of SCI-induced neurogenic bladder, a common consequence of SCI, is not known.

Symptoms of neurogenic bladder include urinary frequency, urgency, and severe urinary incontinence (19). Some studies have confirmed that electroacupuncture treatment has a reparative effect on suprasacral SCI (20). In this paper, the histomorphology of bladder cells and the expression of HCN channel proteins in ICCs, along with the possible functional role of HCN channels, were investigated using an SCI-induced neurogenic bladder rat model. Furthermore, the effects of electroacupuncture at different acupoints on suprasacral SCI were also evaluated.

We present the following article in accordance with the ARRIVE reporting checklist (available at http://dx.doi. org/10.21037/apm-20-1827).

\section{Methods}

\section{Animals}

A total of 120 Sprague Daley (SD) adult male rats weighing 180-220 g at 2 to 3 months of age were purchased from Trophic Laboratory Animal Center. The animals appeared healthy, and were divided into 4 experimental groups described below. All surgical and care procedures administered to the animals were in accordance with the institutional guidelines of the second affiliated Hospital of Wenzhou Medical University and were approved by the ethics committee for research on laboratory animal use of the institution (No. 2019JG112).

\section{Suprasacral SCI model}

In order to establish the suprasacral SCI model, a MASCIS Impactor was used to deliver graded reproducible spinal cord contusions in rats (T8 vertebral body, $2.5 \mathrm{~cm} \times 10 \mathrm{~kg}$ ). Immediately after SCI was induced, the rats were in a state of spinal cord shock. Within 24 hours after the procedure, the bladder was exposed to the bulging upper edge of the pubic symphysis. Postoperative care included antiinfection therapy within 3 days after surgery, daily $0.25 \mathrm{~mL}$ amikacin injected into the thigh muscle of rats, single cage feeding, supplying warmth and nutrition, and postoperative daily massage of rat abdomens to assist in urination, 3 times daily (early, middle and late).

\section{Experimental groups and protocols}

Rats were divided into 4 experimental groups. In the sham 
group, the back skin was cut to expose the T7-T9 vertebral body, and was sutured after 5 minutes without any other treatment. In the SCI model group, rats were operated on as described in 2.2, without any further treatment. The Ciliao group was operated on as described in 2.2. Electroacupuncture treatment commenced3 days after the SCI model was established, and Ciliao and Zhongliao acupoints were selected for continuous treatment for 10 days. In the Guanyuan group, rats were operated on as described in 2.2. Electroacupuncture treatment commenced 3 days after the SCI model was established, and Guanyuan and Zhongji acupoints were selected for continuous treatment for 10 days.

\section{Electroacupuncture treatment}

Disposable aseptic acupuncture needles (Huatuo, Yangzhou Zhixiang Medical Co., Ltd., China) with a specification of $0.18 \times 25 \mathrm{~mm}$ were chosen. All acupoints are referred to in Experimental Acupuncture and Moxibustion (20). Because there are only 3 pairs of posterior sacral foramen in rats, and the S2 nerve controls voiding function, we considered the S2 posterior sacral foramen equivalent to the Ciliao and Zhongliao human acupoints. A filiform needle was inserted $15 \mathrm{~mm}$ deep into the second posterior sacral hole, and the left and right needles were connected for electroacupuncture. The Guanyuan point is located approximately $25 \mathrm{~mm}$ below the umbilical cord (the intersection of $3 / 5$ and $2 / 5$ above the line between the umbilical cord and the superior margin of the pubic symphysis) of the rat. The Zhongii point is located on the anterior midline of the lower abdomen, at the intersection of the upper $4 / 5$ and the lower $1 / 5$ of the line between umbilicus and the superior margin of pubic symphysis, a $5 \mathrm{~mm}$ needle was inserted into Zhongji point. Guanyuan and Zhongji points were connected for electroacupuncture. The parameters for electrical stimulation were continuous pulse current, frequency $2 \mathrm{~Hz}$ and intensity $0.5 \mathrm{~mA}$, using an acupoint stimulator (HANS, Yangzhou Zhixiang Medical Co., Ltd., China). The treatment time of electroacupuncture was 20 minutes each time, once a day, for a total of 10 times.

\section{Hematoxylin and eosin (H\&E) staining}

After electroacupuncture treatment, the rats were intraperitoneally injected with sodium pentobarbital $(80 \mathrm{mg} / \mathrm{kg})$, and the bladder was quickly dissected by laparotomy. A $1 \mathrm{~cm} \times 1 \mathrm{~cm}$ tissue block was taken from the bladder neck, bladder body and bladder triangle. Paraformaldehyde (4\%) was used to fix the tissue at room temperature for 24-48 hours. Tissue was then embedded in paraffin, sliced, stained with $\mathrm{H} \& \mathrm{E}$ and observed under a light microscope. Bladder tissue morphology and computer images were acquired using a $\mathrm{Bx}-70$ microscopic imaging system (Olympus Corporation, Japan).

\section{Western blot}

Rats were sacrificed by intraperitoneal injection of sodium pentobarbital $(80 \mathrm{mg} / \mathrm{kg})$. The bladder was quickly removed from the ice surface, frozen in liquid nitrogen, then stored at $-80{ }^{\circ} \mathrm{C}$ until use. Protein lysates were extracted to obtain cytoplasmic proteins and nuclear proteins, and to detect HCN protein expression in ICCs. The extracted total protein sample was taken out from $-80{ }^{\circ} \mathrm{C}$ and immediately placed on ice (to prevent protein degradation) to thaw. According to the results of protein quantification, a corresponding volume of the total protein sample was added to the protein gel electrophoresis loading buffer, gently mixed, denatured at $95{ }^{\circ} \mathrm{C}$ for 10 minutes, and immediately placed on ice for use. Samples were gently added to the gel wells, the electrophoresis device was set to a steady state, and the voltage was adjusted to $80 \mathrm{~V}$ to pass the sample through the concentrated gel and the separation gel (voltage approximately $8 \mathrm{~V} / \mathrm{cm}$ ). Electrophoresis brings the dye to the proper position of the separation gel thus ending the electrophoresis. After gel electrophoresis, the separated protein bands were transferred to a PVDF membrane through transfer electrophoresis. This experiment used a semi-dry transfer film method. Membranes were then incubated with a non-labeled primary antibody and a horseradish peroxidase-labeled secondary antibody (Abcam Biotechnology Co., Ltd., USA). At room temperature, the second antibody labeled with fluorescein was incubated at room temperature for $1 \mathrm{~h}$. After washing the film for 3 times, ECL chromogenic agent was used to expose the film, and the film scanning instrument was used for imaging.

\section{Real-time PCR}

The tissue block was removed from the liquid nitrogen tank and placed in an ice-cold mortar. Total RNA was extracted according to the Trizolkit instructions (Abcam Biotechnology Co., Ltd., USA). The upstream and downstream primers were designed with reference to the 
Table 1 Primers used for real-time PCR

\begin{tabular}{llc}
\hline $\begin{array}{l}\text { Gene } \\
\text { name }\end{array}$ & Sequence & $\begin{array}{c}\text { Product length } \\
\text { (base pairs) }\end{array}$ \\
\hline HCN1 & F:5'-TTCTTCACAGAGCAAACAA-3' & 99 \\
& R:5'-AGTCCCAGTCCTAAAATTC-3' & \\
HCN2 & F:5'-CAACTTCAACGAGGTGCT-3' & 94 \\
& R:5'-GTTCTTCTTGCCTATGCG-3' & \\
HCN3 & F:5'-ATGCTGACCCCAGTTTCG-3' & 106 \\
& R:5'-TACATCTTCCTGCCCACG-3' & 111 \\
HCN4 & F:5'-TTGCGTTTTGAGGTCTT-3' & \\
& R:5'-TCCTTGTTGCCCTTAGTG-3' & \\
$\beta$-Actin & F:5'-CCTAGACTTCGAGCAAGAGA-3' & 140 \\
& R:5'-GGAAGGAAGGCTGGAAGA-3' & \\
\hline
\end{tabular}

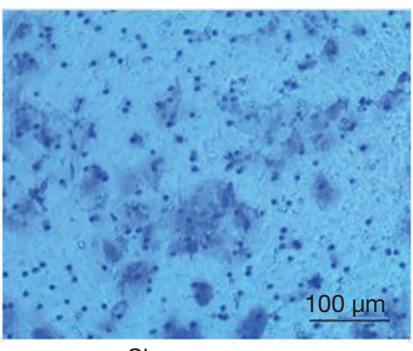

Sham group

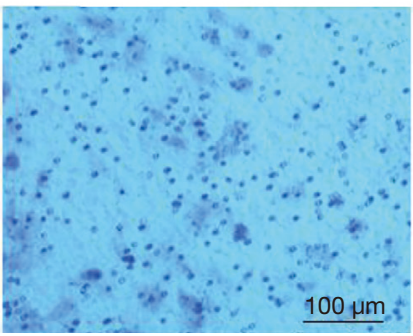

Ciliao group

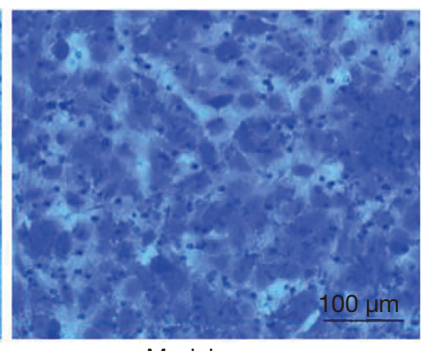

Model group

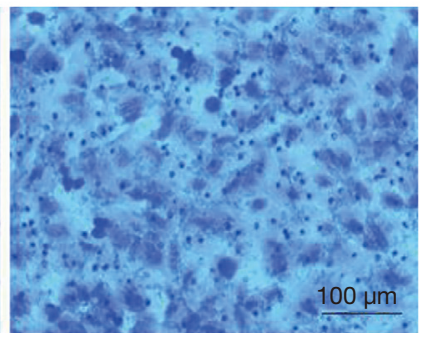

Guanyuan group
Figure 1 Bladder sections with $\mathrm{H} \& \mathrm{E}$ staining at $\times 200$ magnification, marked hypertrophy of muscle layers, an incrassated urothelium and enriched mesenchyme were observed after SCI. SCI, spinal cord injury.

human target genes and housekeeping gene sequences in NCBI Gene bank. The template cDNA was obtained by reverse transcription, and the kinetic curve was determined by fluorescence quantitative PCR. The gene Ct value was compared with the housekeeping gene Ct value, and the relative copy number of the target gene was measured to detect HCN mRNA expression in ICCs. The primer sequences of the target genes are shown in Table 1.

\section{Immunofluorescence}

Bladder tissue sections were fixed in $4 \%$ paraformaldehyde at $4{ }^{\circ} \mathrm{C}$ for 10 minutes. Sections were blocked in $1 \%$ BSA at $37{ }^{\circ} \mathrm{C}$ for 1 hour, then permeabilized using $0.3 \%$ TritonX-100 at room temperature for 10 minutes. After washing 3 times with PBS, sections were incubated with goat anti-human HCN primary antibody (Abcam Biotechnology Co., Ltd., USA) overnight at $4{ }^{\circ} \mathrm{C}$. Sections were then double-labeled with a TRITC and FITC fluorescent secondary antibody (1:150) and incubated at $37^{\circ} \mathrm{C}$ for 1 hour in the dark. DAPI was applied for 2 minutes, then washed with PBS. Sections were inverted for phase contrast fluorescence microscopy (CX33, Olympus Corporation, Japan).

\section{Statistical analysis}

SPSS statistical software (version 22.0, Chicago, IL, USA) was used for statistical analysis. Data was expressed as mean \pm SD. Statistical analysis was conducted using a Student's $t$ test or one-way ANOVA. $\mathrm{P}<0.05$ was considered statistically significant.

\section{Results}

\section{Bladder morphology and histological changes after SCI}

As shown in Figure 1, Bladder histomorphology of each group was observed under light microscopy. In the sham operated group, the epithelial cells were neatly arranged, the intrinsic membrane was intact, and there was no inflammation or edema. The shapes of the detrusor cells were uniform, the muscle fibers were arranged in an orderly manner, the muscle bundles were closely structured, and the connective tissue was filled with gaps. In contrast, the SCI model group had a significantly greater number and level of detrusor fibers compared to the sham group $(\mathrm{P}<0.05)$. At the same time, the muscle fibers were hypertrophied, the level and number of epithelial cells increased, and there was bleeding near the lamina propria and mucosa. Some cell structures and muscle layers were destroyed, and the subgranular layer was seen on the surface of mast cell granules. In the Ciliao group, the number and level of detrusor fibers were also slightly increased, as well as the level and number of epithelial cells $(\mathrm{P}<0.05)$. A small 

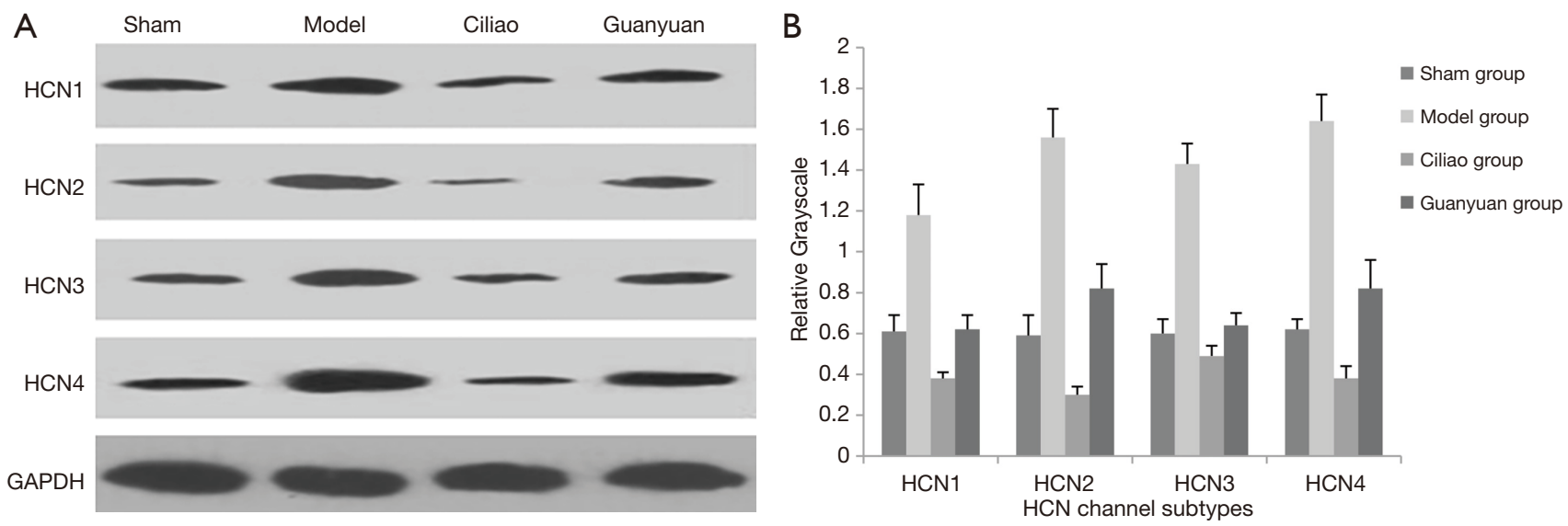

Figure 2 Expression of HCN channel protein in ICCs of the bladder after SCI. After SCI injury, the expression of the HCN channel proteins in the SCI model group were significantly higher compared to all other groups $(\mathrm{P}<0.05)$. Furthermore, the ability of the Ciliao group to inhibit HCN protein expression after injury was significantly lower than that of the Guanyuan group $(\mathrm{P}<0.05)$. HCN, hyperpolarization-activated cyclic nucleotide-gated; GAPDH, glyceraldehyde-3-phosphate dehydrogenase; ICC, interstitial cells of Cajal; SCI, spinal cord injury.

amount of bleeding was observed near the lamina propria and mucosa, and a few cell structures were destroyed. Certain granular layers were visible in the muscular layer and subserosal layer. In the Guanyuan group, the number and level of detrusor fibers increased slightly, and the level and number of epithelial cells increased significantly $(\mathrm{P}<0.05)$. There was significant bleeding near the lamina propria and mucosa. A few cell structures were destroyed, and some granular hypertrophy was seen in the muscular layer and subserosal layer (Figure 1).

\section{Changes in protein expression levels of HCN channels in rat bladder tissue measured by Western blotting}

The expression levels of the 4 subtypes of the HCN channel were similar across the 4 experimental groups $(\mathrm{P}<0.05)$ (Figure 2A). After SCI injury, the expression of the HCN channel proteins in the SCI model group were significantly higher compared to all other groups $(\mathrm{P}<0.05)$. Furthermore, the ability of the Ciliao group to inhibit HCN protein expression after injury was significantly higher than that of the Guanyuan group $(\mathrm{P}<0.05)$ (Figure $2 B)$.

\section{Changes in mRNA expression levels of HCN channels in rat bladder tissue measured by real-time PCR}

According to the original detection result of real-Time PCR and the relative quantitative calculation formula, calculate the relative quantification of the target gene of each sample. As shown in Figure 3, after SCI injury, the expression of the HCN channel mRNA in the SCI model group were significantly higher compared to all other groups $(\mathrm{P}<0.05)$. Furthermore, the ability of the Ciliao group to inhibit HCN mRNA expression after injury was significantly higher than that of the Guanyuan group $(\mathrm{P}<0.05)$.

\section{Immunofuorescence staining reveals changes in protein expression levels of HCN channels in rat bladder tissue after SCI}

Immunofluorescence double labeling of HCN channel protein expression was consistent with the results described above. In the SCI model group, HCN protein expression was significantly higher compared to the other 3 groups. Furthermore, the reduction of HCN protein expression in the Ciliao group after injury was significantly higher than the Guanyuan group (Figure 4).

\section{Discussion}

At present, although the sequelae caused by SCI cannot be completely cured, great progress has been made towards improving the symptoms of SCI and promoting functional repair. The combination of acupoint stimulation and electrical stimulation-electroacupuncture has been widely used in acupuncture and moxibustion, and has achieved 


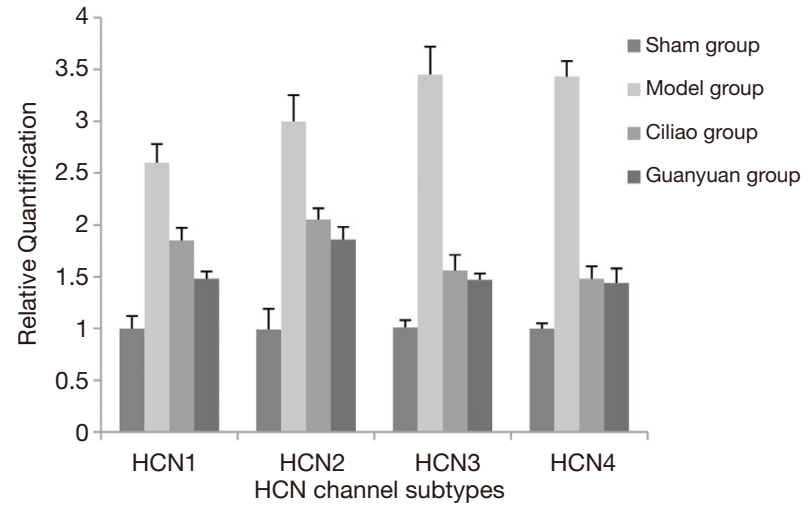

Figure 3 The expression of HCN channel mRNA in ICCs of the bladder after SCI. After SCI injury, the expression of the HCN channel mRNA in the SCI model group were significantly higher compared to all other groups $(\mathrm{P}<0.05)$. Furthermore, the ability of the Ciliao group to inhibit HCN mRNA expression after injury was significantly higher than that of the Guanyuan group $(\mathrm{P}<0.05)$. $\mathrm{HCN}$, hyperpolarization-activated cyclic nucleotide-gated; ICC, interstitial cells of Cajal; SCI, spinal cord injury. good therapeutic effect (21). The principles of acupoint selection for acupuncture prescription include near acupoint selection, far acupoint selection, local acupoint selection and meridian acupoint selection. In the present study, we confirmed that early acupoint electroacupuncture treatment can reduce secondary injury of SCI, and later treatment can reduce the incidence of complications, which is conducive to the functional repair of SCI.

Many studies have shown that after SCI the frequency of bladder detrusor contraction is unstable, the maximum pressure of detrusor is decreased, the number of bladder ICC, the number of HCN channels and the expression of HCN mRNA and protein are increased (22). It is also well known that electroacupuncture can effectively reduce the unstable contraction frequency of the bladder detrusor and the maximum pressure of the detrusor in rats during storage. It can also reduce the number of ICCs in the bladder and the number of HCN channels, and reduce HCN mRNA and protein expression in the bladder
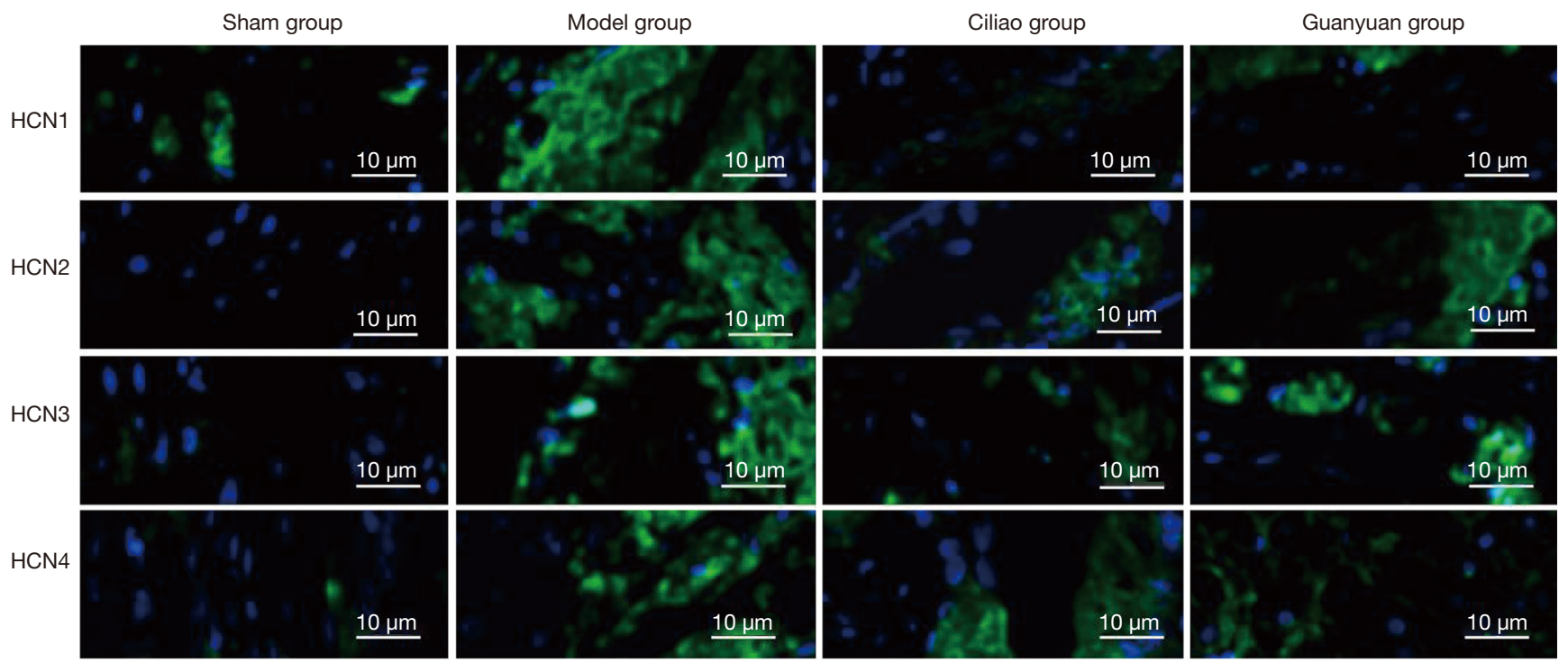

Figure 4 Immunofluorescence staining of bladder tissue across the 4 experimental groups. In bladder ICCs, all 4 HCN subtypes (red) were expressed in all groups. In the SCI model group, HCN protein expression was significantly higher compared to the other 3 groups. Furthermore, the reduction of HCN protein expression in the Ciliao group after injury was significantly higher than the Guanyuan group. HCN, hyperpolarization-activated cyclic nucleotide-gated; ICC, interstitial cells of Cajal; SCI, spinal cord injury. 
detrusor (23). Studies have shown that ICCs are very likely to be the hub of the excitatory regulation mechanism of the bladder itself (24). They integrate and transmit regulatory signals from nerves and stretch stimuli, initiate pacing current signals originating from HCN channels, and pass through various ion channels. Receptor and intercellular communication deliver excitatory signals to the detrusor. Among them, the HCN channel is key, however many ion channels related to calcium ions also participate and play an important role. The HCN channel is widely recognized as a pacing current-initiating channel, and its different subtypes play an important role in cardiac pacing and regulation of the nerve rhythm (25).

Given the known beneficial effects of electroacupuncture on bladder function, and the importance of HCN channels in regulating these functions, our study investigated the expression of HCN channels in bladder ICCs in an SCIinduced neurogenic bladder rat model. A comparison of the sham group and the SCI model group demonstrated that SCI induced significant increases in bladder cells, and destruction of tissue structures in some cells. However, when comparing the SCI model group with the 2 electroacupuncture treatment groups, it was clearly seen that electroacupuncture treatment alleviated this damage. There were also differences between the 2 treatment groups. The ability of the Ciliao group to mitigate SCIinduced histomorphological damage was greater than that of the Guanyuan group.

Furthermore, compared to the sham group, protein expression of the 4 subtypes of the HCN channel after SCI was significantly upregulated in the SCI model group. Expression of all $4 \mathrm{HCN}$ channels after electroacupuncture treatment in the 2 treatment groups was lower compared to the SCI model and sham groups, further confirming the beneficial effect of electroacupuncture on SCI. Between the 2 treatment groups, the Ciliao group demonstrated greater inhibition of HCN channel protein expression after SCI compared to the Guanyuan group. The results of real-time PCR and immunofluorescence analysis were also consistent with the above experimental results.

In conclusion, electroacupuncture treatment had a significant therapeutic effect on an SCI-induced neurogenic bladder rat model. Furthermore, the Ciliao acupoint was superior to the Guanyuan point in alleviating damage after SCI.

\section{Acknowledgments}

Funding: None

\section{Footnote}

Reporting Checklist: The authors have completed the ARRIVE reporting checklist. Available at http://dx.doi. org/10.21037/apm-20-1827

Data Sharing Statement: Available at http://dx.doi. org/10.21037/apm-20-1827

Conflicts of Interest: All authors have completed the ICMJE uniform disclosure form (available at http://dx.doi. org/10.21037/apm-20-1827). The authors have no conflicts of interest to declare.

Ethical Statement: The authors are accountable for all aspects of the work in ensuring that questions related to the accuracy or integrity of any part of the work are appropriately investigated and resolved. All surgical and care procedures administered to the animals were in accordance with the institutional guidelines of the second affiliated Hospital of Wenzhou Medical University and were approved by the ethics committee for research on laboratory animal use of the institution (No. 2019JG112).

Open Access Statement: This is an Open Access article distributed in accordance with the Creative Commons Attribution-NonCommercial-NoDerivs 4.0 International License (CC BY-NC-ND 4.0), which permits the noncommercial replication and distribution of the article with the strict proviso that no changes or edits are made and the original work is properly cited (including links to both the formal publication through the relevant DOI and the license). See: https://creativecommons.org/licenses/by-nc-nd/4.0/.

\section{References}

1. Michael FM, Mohapatra AN, Venkitasamy L, et al. Contusive spinal cord injury up regulates mu-opioid receptor (mor) gene expression in the brain and down regulates its expression in the spinal cord: possible implications in spinal cord injury research. Neurol Res 
2015;37:788-96.

2. Osunronbi T, Sharma H. International Standards for Neurological Classification of Spinal Cord Injury: factors influencing the frequency, completion and accuracy of documentation of neurology for patients with traumatic spinal cord injuries. Eur J Orthop Surg Traumatol 2019;29:1639-48.

3. McGuire EJ, Savastano JA. Long-term followup of spinal cord injury patients managed by intermittent catheterization. J Urol 1983;129:775-6.

4. Derakhshanrad N, Saberi H, Yekaninejad MS, et al. Granulocyte-colony stimulating factor administration for neurological improvement in patients with postrehabilitation chronic incomplete traumatic spinal cord injuries: a double-blind randomized controlled clinical trial. J Neurosurg Spine 2018;29:97-107.

5. Shafik A, Gamal el-Din MA, el-Sibaei O, et al. Involuntary action of the external anal sphincter. Manometric and electromyographic studies. Eur Surg Res 1992;24:188-96.

6. Shafik A. Detrusor sphincter dyssynergia syndrome. A new syndrome and its treatment by external sphincter myotomy. Eur Surg Res 1990;22:243-8.

7. Zhang M, Wang L, Huang S, et al. MicroRNA-223 targets NLRP3 to relieve inflammation and alleviate spinal cord injury. Life Sci 2020;254:117796.

8. Hosseini M, Sarveazad A, Babahajian A, et al. Effect of vitamins $\mathrm{C}$ and $\mathrm{E}$ on recovery of motor function after spinal cord injury: systematic review and meta-analysis of animal studies. Nutr Rev 2020;78:465-73.

9. Calvano CJ, Moran ME, Parekh A, et al. Laparoscopic augmentation cystoplasty using the novel biomaterial Surgisis: small-intestinal submucosa. J Endourol 2000;14:213-7.

10. Rolle U, Piaseczna-Piotrowska A, Puri P. Interstitial cells of Cajal in the normal gut and in intestinal motility disorders of childhood. Pediatr Surg Int 2007;23:1139-52.

11. Jung S, Bullis JB, Lau IH, et al. Downregulation of dendritic HCN channel gating in epilepsy is mediated by altered phosphorylation signaling. J Neurosci 2010;30:6678-88.

12. Hung SW, Marrache S, Cummins S, et al. Defective hCNT1 transport contributes to gemcitabine chemoresistance in ovarian cancer subtypes: overcoming transport defects using a nanoparticle approach. Cancer Lett 2015;359:233-40.
13. Verkerk AO, Wilders R. Pacemaker activity of the human sinoatrial node: an update on the effects of mutations in $\mathrm{HCN} 4$ on the hyperpolarization-activated current. Int J Mol Sci 2015;16:3071-94.

14. Ludwig A, Budde T, Stieber J, et al. Absence epilepsy and sinus dysrhythmia in mice lacking the pacemaker channel HCN2. EMBO J 2003;22:216-24.

15. Miyake S, Higuchi H, Honda-Wakasugi Y, et al. Locally injected ivabradine inhibits carrageenan-induced pain and inflammatory responses via hyperpolarization-activated cyclic nucleotide-gated (HCN) channels. PLoS One 2019; 14:e0217209.

16. He JT, Li XY, Zhao X, et al. Hyperpolarizationactivated and cyclic nucleotide-gated channel proteins as emerging new targets in neuropathic pain. Rev Neurosci 2019;30:639-49.

17. Boulton S, Akimoto M, Akbarizadeh S, et al. Free energy landscape remodeling of the cardiac pacemaker channel explains the molecular basis of familial sinus bradycardia. J Biol Chem 2017;292:6414-28.

18. Won J, Lee PR, Oh SB. Alpha 2 adrenoceptor agonist guanabenz directly inhibits hyperpolarizationactivated, cyclic nucleotide-modulated (HCN) channels in mesencephalic trigeminal nucleus neurons. Eur J Pharmacol 2019;854:320-7.

19. Faerman I, Maler M, Jadzinsky M, et al. Asymptomatic neurogenic bladder in juvenile diabetics. Diabetologia 1971;7:168-72.

20. Fowler CJ, Jewkes D, McDonald WI, et al. Intravesical capsaicin for neurogenic bladder dysfunction. Lancet 1992;339:1239.

21. Kirshblum S, Snider B, Rupp R, et al. Updates of the International Standards for Neurologic Classification of Spinal Cord Injury: 2015 and 2019. Phys Med Rehabil Clin N Am 2020;31:319-30.

22. Brennan GP, Baram TZ, Poolos NP. HyperpolarizationActivated Cyclic Nucleotide-Gated (HCN) Channels in Epilepsy. Cold Spring Harb Perspect Med 2016;6:a022384.

23. Akimoto M, Zhang Z, Boulton S, et al. A mechanism for the auto-inhibition of hyperpolarization-activated cyclic nucleotide-gated (HCN) channel opening and its relief by cAMP. J Biol Chem 2014;289:22205-20.

24. Si X, Huang L, Gong Y, et al. Role of calcium in activation of hyperpolarization-activated cyclic nucleotide-gated channels caused by cholecystokinin octapeptide in 
interstitial cells of cajal. Digestion 2012;85:266-75.

25. Sartiani L, Mannaioni G, Masi A, et al. The

Hyperpolarization-Activated Cyclic Nucleotide-Gated

Channels: from Biophysics to Pharmacology of a Unique
Family of Ion Channels. Pharmacol Rev 2017;69:354-95.

(English Language Editor: C. Belazar-Maseh)

Cite this article as: Lu JY, Ying XW, Chen XL, Tu WZ, Li SS, Jiang SH. Effects of electroacupuncture at different acupoints on the histomorphology of neurogenic bladder and the expression of hyperpolarization-activated cyclic nucleotidegated channels in interstitial cells of Cajal in a rat model of suprasacral spinal cord injury. Ann Palliat Med 2020;9(6):3830-3838. doi: 10.21037/apm-20-1827 